\title{
Concepts of modeling surface energy anisotropy in phase-field approaches
}

\author{
Oleg Tschukin ${ }^{*}$, Alexander Silberzahn ${ }^{1}$, Michael Selzer ${ }^{1,2}$, Prince G. K. Amos ${ }^{1}$, Daniel Schneider ${ }^{1,2}$ \\ and Britta Nestler ${ }^{1,2}$
}

*Correspondence:
oleg.tschukin@kit.edu
${ }^{1}$ Institute for Applied
Materials (IAM-CMS),
Karlsruhe Institute
of Technology, Building
30.48, Strasse am Forum 7,
76131 Karlsruhe, Germany
Full list of author information
is available at the end of the
article

${ }^{*}$ Correspondence: oleg.tschukin@kit.edu ${ }^{1}$ Institute for Applied Materials (IAM-CMS), of Technology, Building 30.48, Strasse am Forum 7 Full list of author information article

\begin{abstract}
To simulate the growth of geological veins, it is necessary to model the crystal shape anisotropy. Two different models, classical and natural models, which incorporate the surface energy anisotropy into the objective functional of Ginzburg-Landau type, are presented here. Phase-field evolution equations, considered in this work, are derived using the variational approach, and correspond to the conservative Allen-Cahn-type equation. For three characteristic anisotropy formulations, we show what kind of difficulties arise in the simulations for the presented models. Particularly, if the anisotropy becomes strong, the phase-field evolution equations become ill-posed. Thus, we present regularized phase-field models and discuss the corresponding simulation results. Furthermore, in the scope of the grain growth simulation, we extend the original twophase models to multiphases.
\end{abstract}

Keywords: Phase field, Anisotropy, Allen-Cahn equation

\section{Background}

In the field of geothermal energy, new developing and integrating technologies are necessary for its establishment as a key element in the future global energy concept. In the field of engineering, the realization of novel projects which use geothermal energy requires years of practical experience in geology, geophysics, and geochemistry. Furthermore, there is no exact knowledge about the relevant natural and technological processes taking place, and thus, the real understanding of these processes is incomplete.

The basic working principle of the geothermal power plants, as the most common way of capturing the energy from geothermal sources, is based on hydrothermal convection. Meanwhile, cool water is infiltrated into the Earth's crust, where it penetrates through the rock cavities and is heated up at a depth of several hundred meters. Then, the now warmer water/steam rises to the surface, wherein the heat power drives the electric generator. Saturating the fluid with salts improves its thermal properties, but enhances an undesired cleft sealing due to the vein growth on the walls of the rock cavities. Thus, finding the balance between these aspects is of special interest. Extensive research is needed to optimize the process parameters, but with a manageable economical effort.

In natural sciences, the laboratory experiments usually serve to emphasize the relevant connections between the significant process quantities and form a base for the

(c) The Author(s) 2017. This article is distributed under the terms of the Creative Commons Attribution 4.0 International License (http://creativecommons.org/licenses/by/4.0/), which permits unrestricted use, distribution, and reproduction in any medium, provided you give appropriate credit to the original author(s) and the source, provide a link to the Creative Commons license, and indicate if changes were made. 
theories and new technologies. However, the geothermal processes in the Earth's crust are difficult to recreate in the laboratory, because of enormous dimensions, the lengthy period of processes and the unknown but estimated physical and chemical conditions and material parameters. Thus, alternative methods are required to mimic the phenomena in the Earth's crust to understand the geothermal processes for new technological development.

Nowadays, in the epoch of high-performance computers, the real geological, geophysical, and geochemical processes occurring in the Earth's crust can be reproduced individually as well as collectively in virtual experiments-simulations. However, in contrast to the real processes subjected to natural laws, the virtual experiments are based on the human understanding of these natural laws, generally expressed in mathematical models. By validating the simulation results through a comparison with natural states, the mathematical models are able to predict complex interactions, and may be used as novel technology to control and manipulate natural processes.

During vein growth in the clefts of the Earth, a variety of geological conditions, such as the local deformation rates and the cleft surface texture, as well as the degree of salt supersaturation and the velocity of the solution, to mention a few, influence the evolution of veins, and thus reveal the high complexity of the featured process. Note: the more complex the analyzed system is, the more unmanageable the corresponding mathematical model for the computational experiments becomes.

A phase-field model is one of the potential methods which meets the required challenges that arise during the modeling and simulation of collective vein growth. In the context of the crack opening and crack sealing history, as well as in dependence of the surface texture, three-dimensional simulations of the vein evolution in unitaxial (Ankit et al. 2013) and bitaxial quartz (Ankit et al. 2015a, b) veins are presented and discussed in the doctoral thesis by Ankit (2016). Within the in situ solver PACE3D, Selzer (2015) demonstrated a numerical study of fluid flow in the Earth's clefts, using the Darcy law as well as the Navier-Stokes model. Although simplified assumptions are used in these works, by performing virtual experiments, they form a milestone for forthcoming works by simulating geological structures, and by incorporating geochemical, geothermal, geophysical, and technological conditions.

Since their inception, the phase-field models have progressively proven to be an efficient numerical tool for the simulation of dynamic systems involving moving interfaces (Chen 2002). Often, the interface which separates different sub-volumes varies depending on the system. During solidification, for instance, the interface is a common surface, distinguishing the growing crystal from its liquid (Kobayashi 1993; Karma and Rappel 1998), whereas in solid-state transformation (Schneider et al. 2016), it is the boundary between the two crystals of different crystal structures, orientation, or composition. The grain growth in a supersaturated environmental solution is a diffusion-reactiondriven process, and is a further example in which the growth process is mathematically described by the time-dependent free boundary problem, coupled with the macroscopic processes. Thus, although originally confined to the simulation of the microstructural evolution (Plapp and Karma 2002; Steinmetz et al. 2016), the flexibility of the phase-field 
method extends its application to several avenues of research, like geology (Ankit 2016), medicine and biology (Emmerich and Travasso 2011; Garcke et al. 2016), topology optimization (Selzer 2015), and others.

To model and simulate vein growth, and, actually, to accommodate the various structures, shape anisotropy is introduced into the model. A pioneering attempt to incorporate anisotropy is seen in the work of Kobayashi (1993), where dendritic structures are simulated. Here, the anisotropy is incorporated into the model by the interfacial energy, and the diffuse interface width between two transient phases is orientation-dependent. Furthermore, the interfacial energy in the diffuse interface exactly meets the directiondependent interfacial energy in the sharp interface limit. This claim, which has found a broad approval in the phase-field community, is known as a classical approach and warrants the extension of the original two-phase model to multiphase-field models (Steinbach et al. 1996; Garcke et al. 1998; Nestler et al. 2005).

Alternative phase-field models with anisotropy are presented by Kobayashi et al. (1998), Eggleston et al. (2001), Ma et al. (2006), Fleck et al. (2011), Torabi et al. (2008), Miranville (2015), Makki (2016), to mention a few representative works. While in the former model (Kobayashi et al. 1998), an orientation field is introduced to differentiate anisotropic formations (Takaki 2014), we disregard this kind of model and refer an interested reader to Tóth et al. (2015); Korbuly et al. (2017). The latter models (Eggleston et al. 2001; Ma et al. 2006; Fleck et al. 2011; Torabi et al. 2008; Miranville 2015; Makki 2016) only deal with phase field and are particularly discussed in this work.

For all approaches, the phase-field evolution equations are rooted in a variational formulation, but the objective functional and the consistently moving equations vary from model to model. In this work, we give a concise, yet substantial overview of the distinctions between the different concepts of modeling surface energy anisotropy within the phase-field method. Therefore, the present manuscript is of academic interest for the modeling and simulation community, with a particular focus on the field of geothermal energy, while proposing appropriate methods to simulate the growth of salts in the Earth's crust.

The unfolding of this work begins with a general formulation of the phase-field approach, comprising useful properties of the isotropic model. Next, the proposed anisotropies are defined and then incorporated into two different objective functionals of Ginzburg-Landau type. Thus, the classical and natural models differ in the formulations and in the corresponding moving equations. For the special anisotropy functions, for which the surface stiffness becomes negative, an additional term is incorporated into the functional to regularize the evolution equations. The extended models are referred to as regularized classical and regularized natural models. The simulation results corresponding to the defined anisotropy functions are elucidated in the subsequent section, which are then comprehensively discussed in the following paragraph. Then, an outlook of the presented approaches to multiphase systems is provided to emphasize its significance for the collective grain growth simulations. In the last section, we conclude the results presented in this work. 


\section{Phase-field model}

The fundamental concept governing all phase-field simulations is the temporal evolution of an optimized Lyapunov functional, which, based on the formulation, either remains constant, increases monotonically (Garcke et al. 1998; Nestler et al. 2005), or decreases (Cahn and Hilliard 1958) to an extremum. One such formulation is chosen for the present study, where the non-increasing free energy functional of Ginzburg-Landau type (Landau and Lifschitz 1992), $\partial_{t} \mathcal{F} \leq 0$, monotonically converges to its minimum

$$
\mathcal{F}[\boldsymbol{\phi}, \boldsymbol{s}]=\int_{V} \epsilon a(\boldsymbol{\phi}, \nabla \boldsymbol{\phi})+\frac{1}{\epsilon} w(\boldsymbol{\phi}, \nabla \boldsymbol{\phi})+f(\boldsymbol{\phi}, \boldsymbol{s}) \mathrm{d} V .
$$

This formulation introduces the phase-field vector $\phi=\left(\phi_{1}, \phi_{2}, \ldots, \phi_{N}\right)$, an element of the Gibbs simplex

$$
\mathcal{G}=\left\{\phi \in \mathbb{R}^{N} \mid \phi_{\alpha} \in[0,1], \quad \sum_{\alpha=1}^{N} \phi_{\alpha}=1\right\},
$$

which represents the $N$-constituent phases.

As we consider equilibrium shapes and no growth processes, we neglect the third term in the functional $(f(\phi, s)=0)$, which originally depends on other macroscopic state variables, $\boldsymbol{s}$, which define the driving forces of phase transformations. Thus, the functional simplifies to the form

$$
\mathcal{F}[\phi]=\int_{V} \epsilon a(\phi, \nabla \phi)+\frac{1}{\epsilon} w(\phi, \nabla \phi) \mathrm{d} V,
$$

where $\epsilon a(\boldsymbol{\phi}, \nabla \boldsymbol{\phi})$ and $\frac{1}{\epsilon} w(\boldsymbol{\phi}, \nabla \boldsymbol{\phi})$ denote the gradient energy and the potential, respectively. Evidently, both functions are dependent on the phase field $\phi=\left(\phi_{1}, \ldots, \phi_{N}\right)$ as well as on its gradient $\nabla \boldsymbol{\phi}=\left(\nabla \phi_{1}, \ldots, \nabla \phi_{N}\right)$.

The phase-field variables involved in this formulation vary in their type, depending on the considered system. In some cases, the $\phi$-vector represents the local volume fraction of the phases, while in others, $\phi$ represents composition, vector-valued orientation, polarization, etc. Hence, there is no "the" phase-field model, but an approach to address a given problem. Despite this flexibility, the proportionality between the thermodynamic driving forces and fluxes, $J_{\phi_{\alpha}} \propto-\frac{\delta \mathcal{F}}{\delta \phi_{\alpha}}$, involving the variational derivative

$$
\frac{\delta}{\delta \phi_{\alpha}}=\frac{\partial}{\partial \phi_{\alpha}}-\nabla \cdot \frac{\partial}{\partial \nabla \phi_{\alpha}}
$$

is assumed.

If the phase-field variables are indicative of the composition or internal energy density, the system variables remain locally conserved. Due to this constraint, and according to the conservation law, the evolution equations of Cahn-Hilliard type are written as follows (Nestler et al. 2005; Eiken et al. 2006; Choudhury and Nestler 2012):

$$
\partial_{t} \phi_{\alpha}=-\nabla \cdot\left(J_{\phi_{\alpha}}-J_{\phi_{N}}\right), \quad \forall i \in\{1, \ldots, N-1\} .
$$

In contrast to conserved variables, the Allen-Cahn-type equation

$$
\partial_{t} \phi_{\alpha}=M J_{\phi_{\alpha}}
$$


describes the evolution for the non-conserved variables, like the orientation field (Kobayashi et al. 1998). This form of the equation changes to

$$
\partial_{t} \phi_{\alpha}=M\left(J_{\phi_{\alpha}}-\lambda\right),
$$

when the local volume fractions $\phi=\left(\phi_{1}, \ldots, \phi_{N}\right)$ are restricted through the local volume constraint $\sum_{\alpha}^{N} \phi_{\alpha}=1$. Here, $\lambda=-\frac{1}{N_{A}} \sum_{\alpha} \frac{\delta F}{\delta \phi_{\alpha}}$ is the appropriate Lagrange multiplier, with $N_{A}$ being the number of locally present phases (Selzer 2015).

A total volume-fraction conservation of the $\alpha$-phase modifies the previous evolution equation to

$$
\partial_{t} \phi_{\alpha}=M\left(J_{\phi_{\alpha}}-\lambda-\frac{\partial g\left(\partial_{t} V_{\alpha}, \phi_{\alpha}\right)}{\partial \phi_{\alpha}}-\Lambda\right),
$$

with an additional anti-driving force, $g\left(\partial_{t} V_{\alpha}, \phi_{\alpha}\right)$, due to the total volume change $\partial_{t} V_{\alpha}$ of the $\alpha$-phase. $\Lambda$ is the respective additional contribution to the Lagrange multiplier $\lambda$. For details of the model as well as of the numerical solving algorithm, see Nestler et al. (2008). Note that for the Cahn-Hilliard Eq. (3) and for the conservative Allen-Cahn equation (5) the evolution paths as well as the equilibrium states could be quite different, as was shown by Lee and Kim (2016).

In the present simulation study, the focus is placed on the constrained form of the Allen-Cahn Eq. (5), as its phase-field represents the local volume fraction of the corresponding phase. Furthermore, the number of phases is confined to two, since the emphasis is on the presentation of different incorporation types of anisotropy into the objective functional. Owing to these considerations, the number of independent variables reduces to one.

\section{Isotropic phase-field model for two phases}

Despite the numerous formulations for multiphase systems with isotropic surface energies (Tóth et al. 2015), the objective functional for a two-phase system is generally written as follows:

$$
\mathcal{F}=\int_{V} \gamma\left(\epsilon|\nabla \phi|^{2}+\frac{1}{\epsilon} w(\phi)\right) \mathrm{d} V
$$

whereby the modeling parameters $\gamma$ and $\epsilon$ relate to the interface energy $\sigma$ and to the interface width $W$. To ensure the correct physics of the setup, the physical interfacial energy $\sigma$ should be reflected exactly in the model. The real physical interface width of the order of nanometers evokes a challenge when its evolution is collectively analyzed with changes in the mesoscopic bulk phases. In the phase-field model, this conflict is removed using a much higher diffuse interface width than the real physical width. Note that both the interfacial energy as well as the diffuse interface width depend on the choice of the potential $w(\phi)$ (Taylor and Cahn 1998).

In contrast to the double well potential, which is mostly used in the phase-field community, the double obstacle potential

$$
w^{o b}(\phi)= \begin{cases}\frac{16}{\pi^{2}} \phi(1-\phi), & \phi \in \mathcal{G} \\ \infty, & \text { else }\end{cases}
$$


contains non-vanishing derivatives for the values $\phi=0$ and $\phi=1$, introducing inadmissible values of $\phi<0$ and $\phi>1$ during the numerical integration. This mathematical property is achieved by the back projection onto the Gibbs simplex in Eq. (2). Thus, the phase-field value, smaller than zero or greater than one, is set to zero or one, respectively. For multiple phases, the Gibbs simplex projection is more complicated, and an interested reader is exemplarily referred to Chen and Ye (2011).

Beneficial for the double obstacle potential is the corresponding one-dimensional equilibrium phase-field profile, which is expressed as follows:

$$
\phi_{e q}^{o b}(x)= \begin{cases}0, & x<-0.125 \pi^{2} \epsilon, \\ 0.5\left(1+\sin \left(\frac{4 x}{\pi \epsilon}\right)\right), & x \in[-0.125,0.125] \pi^{2} \epsilon, \\ 1, & x>0.125 \pi^{2} \epsilon\end{cases}
$$

and which suggests that, at equilibrium, it is dependent on the model parameter: $\epsilon$, and is related to the finite thickness of the diffuse interface by

$$
W=\frac{\pi^{2}}{4} \epsilon
$$

Owing to its governing influence on the finite interface width and its subsequent computational advantage, the double obstacle potential is used in all simulations of the present work. Furthermore, the real interfacial energy $\sigma$ and the modeling parameter $\gamma$ should not be the same (Choudhury and Nestler 2012). However, according to our approach, the isotropic surface energy can be used directly as modeling parameter

$$
\gamma=\sigma
$$

Note that the locus $\phi=0.5$ typically represents the sharp interface contour, since by converging the model parameter $\epsilon$ to zero, the phase-field profile in Eq. (7) attains the Heaviside step function, which represents the sharp interface consideration.

\section{Anisotropic phase-field models for two phases}

Pioneering work in the scope of crystal shapes and the corresponding interface energy was done by Wulff (1901). In his work, Herring (1951) presented an elegant procedure to construct the Wulff shape for a given surface energy anisotropy, $\sigma(\boldsymbol{n})$, which is often represented graphically in a polar plot of $\sigma(\boldsymbol{n}(\theta, \varphi))$ versus the azimuthal and polar angles $\theta$ and $\varphi$ of normal $\boldsymbol{n}$ (Sekerka 2005). In two dimensions, the expression reduces to a function of only one angle $\theta=\arctan \frac{n_{y}}{n_{x}}$, and the corresponding Wulff shape is a convex hull (Mullins 1962) bounded by the perpendiculars to $\boldsymbol{n}$, at a distance $\sigma(\boldsymbol{n})$, from the origin (Sekerka 2005). According to Garcke et al. (1999), the surface energy anisotropy function can be written as follows:

$$
\sigma(\boldsymbol{n})=\sigma_{0} A(\boldsymbol{n})
$$

with the essential dimensionless anisotropy function $A(\boldsymbol{n})$ depending on the unit vector $\boldsymbol{n}=\left(n_{x}, n_{y}\right)^{T}$, normal to the interface, and with constant $\sigma_{0}$.

Depending on the essential anisotropy function, the corresponding Frank plot, defined by $1 / \sigma(\boldsymbol{n})$, indicates the regularity of the Wulff shape construction. For a convex Frank 
plot, the resulting equilibrium shape is well defined (Sekerka 2005). On the other hand, for a non-convex Frank plot, the equilibrium shape consists of the so-called "ears", due to the missing orientations with $\sigma(\theta)+\sigma^{\prime \prime}(\theta)<0$, which do not belong to the convex hull. Equivalently, the Cahn-Hoffman vector (Hoffman und Cahn 1972; Cahn und Hoffman 1974; Wheeler 2006), "which provides a graphical representation of the equilibrium shape in the presence of anisotropic interfaces and their propensity to break up into facets", is not unique-Abdeljawad et al. (2016).

In addition to the shape anisotropy, the kinetic anisotropy plays a crucial role for the crystal growth, as reported particularly by Karma and Rappel (1998), McFadden et al. (1993) and Sekerka (2005). In our work, we only consider shape anisotropy and disregard the kinetic anisotropy.

In the following, we, respectively, introduce and discuss three anisotropy functions of $A(\boldsymbol{n})$ : cubic positive (Kobayashi 1993), elliptical and crystalline functions

$$
\begin{aligned}
& A(\boldsymbol{n})=1-F_{\alpha \beta}\left(3-4\left(n_{x}^{4}+n_{y}^{4}\right)\right) \quad\left(=1+F_{\alpha \beta} \cos (4 \theta)\right) \\
& A(\boldsymbol{n})=I_{\alpha \beta} \cdot n_{x}^{2}+n_{y}^{2} \quad\left(=0.5\left(1+I_{\alpha \beta}+\left(I_{\alpha \beta}-1\right) \cos (2 \theta)\right)\right. \\
& A(\boldsymbol{n})=\max _{1 \leq k \leq K}\left\langle\boldsymbol{n}, \eta^{k}\right\rangle .
\end{aligned}
$$

The expressions in the parentheses are the equivalents written with the polar angle $\theta$.

The parameters $F_{\alpha \beta}$ and $I_{\alpha \beta}$ describe the strength of the anisotropy, which determines the deviation from the unit sphere. For the faceted anisotropy, Eq. (11), the vectors $\eta^{k}$ in Eq. (11) are the corners of the Wulff shape and allow to model arbitrary crystal formations.

For the function $\gamma(\boldsymbol{n})$ in the objective functionals, we use the physical surface energy anisotropy function $\gamma(\boldsymbol{n})=\sigma(\boldsymbol{n})=\sigma_{0} A(\boldsymbol{n})$. Moreover, since the equilibrium shape is independent of the magnitude of $\sigma_{0}$, we assume that $\sigma_{0}=1$ for simplicity. The normal vector components are determined by the phase-field gradient as follows:

$$
\boldsymbol{n}=\left(\begin{array}{l}
n_{x} \\
n_{y}
\end{array}\right)=\frac{\nabla \phi}{|\nabla \phi|}
$$

and thus, the anisotropy function explicitly depends on the phase-field gradient.

There are different approaches to incorporate the anisotropy function into the phasefield model. In Kobayashi (1993), Kobayashi used the model

$$
\mathcal{F}_{1}=\int_{V} \epsilon A^{2}(\boldsymbol{n})|\nabla \phi|^{2}+\frac{1}{\epsilon} w(\phi) \mathrm{d} V
$$

which we refer to as classical model, to simulate dendrites. Note that the anisotropy function is incorporated squared, and solely in the gradient energy term. In this formulation, the diffuse interface width $W(\boldsymbol{n})=W_{0} A(\boldsymbol{n})$ also becomes orientation-dependent. Following this approach, the corresponding variational derivative is written as follows: 


$$
-\frac{\delta \mathcal{F}_{1}}{\delta \phi}=\epsilon \nabla \cdot\left[2 A(\boldsymbol{n})\left(\frac{\partial A(\boldsymbol{n})}{\partial \nabla \phi}|\nabla \phi|^{2}+A(\boldsymbol{n}) \nabla \phi\right)\right]-\frac{1}{\epsilon} w^{\prime}(\phi) .
$$

This model, with various anisotropy formulations, achieved a broad approval in the phase-field community. A detailed analysis of this model was discussed by Taylor and Cahn (1998) and in citations therein.

The dependence of the interface width on anisotropy is averted by introducing the anisotropy function for both gradient energy as well as potential energy (Ma et al. 2006; Torabi et al. 2008; Steinbach 2009; Fleck et al. 2011). Thus, an alternative objective free energy functional can be written as follows:

$$
\mathcal{F}_{2}=\int_{V} A(\boldsymbol{n})\left(\epsilon|\nabla \phi|^{2}+\frac{1}{\epsilon} w(\phi)\right) \mathrm{d} V .
$$

"This is a natural formulation if we interpret the term in the parentheses as approximating the surface delta function"-Torabi et al. (2008), and thus, we call it a natural model. Contrary to the classical model, Eq. (12), the anisotropy function scales the gradient energy and the potential in the formulation. The variational derivative of Eq. (14), for the phase-field evolution, Eq. (5) is written as follows:

$$
-\frac{\delta \mathcal{F}_{2}}{\delta \phi}=\epsilon \nabla \cdot\left[\frac{\partial A(\boldsymbol{n})}{\partial \nabla \phi}\left(|\nabla \phi|^{2}+\frac{1}{\epsilon^{2}} w(\phi)\right)+2 A(\boldsymbol{n}) \nabla \phi\right]-\frac{1}{\epsilon} A(\boldsymbol{n}) w^{\prime}(\phi) .
$$

We want to highlight the difference between the classical and the natural model. Since the anisotropy function in the classical model solely belongs to the gradient energy part and is squared, it is used directly in both gradient energy and potential, in the natural model. Because of these differences in the formulation [Eqs. (12) and (14)], the resulting evolution Eq. (5) are also different due to the respective variational derivative in Eqs. (13) and (15). Nevertheless, if the corresponding Wulff shape is well defined, the interfacial energy in the diffuse interface equates to the sharp interface form $\gamma(\boldsymbol{n})=\sigma(\boldsymbol{n})$, in spite of the model. Furthermore, the orientation-dependent diffuse interface width in the classical model becomes orientation-independent in the natural model (Ghosh et al. 2015).

For smooth surface energies, Eqs. (9) and (10), with $F_{\alpha \beta} \leq 1 / 15$ and $I_{\alpha \beta} \leq 2$, respectively, and for the crystalline anisotropy, Eq. (11), the corresponding Frank plot is convex, and the resulting equilibrium shapes are well defined (Sekerka 2005). For a non-convex Frank plot, $\left(F_{\alpha \beta}>1 / 15\right.$ or $\left.I_{\alpha \beta}>2\right)$, the surface stiffness becomes negative, and the corresponding phase-field problem becomes ill-posed (Taylor and Cahn 1998; Torabi et al. 2008; Miranville 2015).

To correct the aforementioned discrepancy, a clear distinction between well- and illposed problems is necessary. Different approaches were presented in the past to solve the problem of the ill-posedness of the evolution equations. One possibility is to change the formulation of the interface energy $\sigma(\boldsymbol{n}) \rightarrow \sigma^{(c)}(\boldsymbol{n})$ (Taylor and Cahn 1998) to its "convexified" form, but with equivalent Wulff shapes. The technical details of this construction are presented by Eggleston et al. (2001) and applied, in particular, in the phasefield simulations by Fleck et al. (2011). We disregard this approach of the regularization of the anisotropy formulation, but consider the regularization of the phase-field model, 
whereby an additional term is incorporated into the objective functionals [Eqs. (12) and (14)]. Following Wheeler (2006) the free energy functional is rewritten as follows:

$$
\mathcal{F}_{1}^{\text {reg }}=\int_{V} \epsilon A^{2}(\boldsymbol{n})|\nabla \phi|^{2}+\frac{1}{\epsilon} w(\phi)+\frac{B}{\epsilon} H^{2} \mathrm{~d} V,
$$

and according to Torabi et al. (2008), we obtain

$$
\mathcal{F}_{2}^{r e g}=\int_{V} A(\boldsymbol{n})\left(\epsilon|\nabla \phi|^{2}+\frac{1}{\epsilon} w(\phi)\right)+\frac{B}{\epsilon} H^{2} \mathrm{~d} V .
$$

The regularization term $H$ is the mean curvature of the corresponding locus $\phi=$ const and is written as follows:

$$
H=\frac{1}{\epsilon} w^{\prime}(\phi)-\epsilon \Delta \phi
$$

A similar regularization term of the classical model is found by Wheeler (2006) as well as by Ghosh et al. (2015), but with an alternative evaluation of the mean curvature, given by $H=\nabla \cdot \boldsymbol{n}$. Note, furthermore, that the energetics of the interface, originally comprised of gradient energy and potential, is now modified by the incorporation of an additional term. Thus, the contribution of the curvature is interpreted as a higher order term in the power expansion of the surface tension (Torabi and Lowengrub 2012). Obviously, the functionals in Eqs. (16) and (17), with the parameter $B=0$, are without regularization and result in the original formulations in Eqs. (12) and (14), respectively.

Furthermore, the incorporation of the regularization term into the objective functionals changes the variational derivative to

$$
\frac{\delta \mathcal{F}}{\delta \phi}=\frac{\partial \mathcal{F}}{\partial \phi}-\nabla \cdot \frac{\partial \mathcal{F}}{\partial \nabla \phi}+\Delta \frac{\partial \mathcal{F}}{\partial \Delta \phi},
$$

and the original second-order conservative Allen-Cahn Eq. (5) becomes a fourth-order equation for the classical model:

$$
-\frac{\delta \mathcal{F}_{1}^{\text {reg }}}{\delta \phi}=-\frac{\delta \mathcal{F}_{1}}{\delta \phi}-\frac{2 B}{\epsilon}\left(\frac{1}{\epsilon} w^{\prime \prime}(\phi) H-\epsilon \Delta H\right),
$$

and for the natural model:

$$
-\frac{\delta \mathcal{F}_{2}^{\text {reg }}}{\delta \phi}=-\frac{\delta \mathcal{F}_{2}}{\delta \phi}-\frac{2 B}{\epsilon}\left(\frac{1}{\epsilon} w^{\prime \prime}(\phi) H-\epsilon \Delta H\right) .
$$

\section{Numerical experiments}

Simulations rendered in the present work are performed using the solver PACE3D (Parallel Algorithms of Crystal Evolution in 3D), consisting of over 560,000 lines of C code. In this multifunctional solver, the phase-field equations are basically solved in three dimensions in an efficient manner, coupled with physical processes like mass and heat diffusion, fluid flow, elastic and plastic effects, etc. Furthermore, the solver PACE3D is constantly developed and optimized, in accordance with the recent advancements.

The right-hand side of the evolution Eq. (5) is spatially discretized using the finite difference method, optimized for two-dimensional simulations, and the temporal derivative 
on the left-hand side is approximated by the forward difference scheme, known as the explicit Euler scheme. The numerical scheme and the solving algorithm are given in detail by Nestler et al. (2008).

To highlight the differences between the phase-field models, Eqs. (12), (14), (16), and (17), and their applicability for the well- and ill-posed problems, the expected equilibrium shapes (solid magenta lines), constructed due to the given surface energy anisotropies (solid blue lines), are shown in the following figures as polar plots. The ill-posed problems can be recognized by the presence of the "ears" in the Wulff plot, which do not belong to the equilibrium shape. For the predefined smooth anisotropy functions, Eqs. (9) and (10), in the cases with $F_{\alpha \beta} \geq 1 / 15$ and $I_{\alpha \beta} \geq 2$, the surface stiffness becomes negative, and the corresponding Wulff shape with "ears" belongs to these missing orientations. In the following, we use different values of the parameters $F_{\alpha \beta}$ and $I_{\alpha \beta}$, and analyze both well-defined equilibrium shapes and numerical equilibrium shapes for anisotropies with missing orientations.

To invoke comparability in the present work, the corner vectors for the faceted anisotropy, Eq. (11), are restricted to a rhombus, $\eta^{1,2}=( \pm 2,0)^{T}$ and $\eta^{3,4}=(0, \pm 1)^{T}$.

The outcomes of the diffuse interface simulations are accommodated through the locus $\phi=0.5$, represented by thick black lines in the following figures. The applicability of the model is highlighted by overlaying the Wulff shape on the resulting precipitate. All simulations in the current work are performed with an initial filling of a circular inclusion, with a diameter of 50 dimensionless grid cells of $\Delta x=1$.

To resolve the phase-field profile in Eq. (7), the consequential gradient energy and the potential in the simulations accurately, approximately 15 numerical cells, are required for the diffuse interface resolution. A simple algebraic relation between the width $W$ and the model parameter $\epsilon$, in Eq. (8), $(15 \triangle x \approx 2.5 \epsilon)$, leads to a dimensionless value of $\epsilon=6 \triangle x$.

Since we are solely interested in the equilibrium state, but not in the dynamic change of the initial inclusion, the mobility coefficient $M$ in the phase-field evolution equation, Eq. (5), plays the role of a relaxation parameter. By analyzing the kinetic evolution of a crystal growth, the mobility coefficient represents the growth kinetics and should be adopted to the physics. In all the following simulation studies, the mobility is set to $M=1 / \epsilon=1 / 6$. Changing the mobility influences the duration of the convergence to the equilibrium state, but not the final equilibrium shape.

The temporal step is chosen due to the stability criterion

$$
\Delta t=0.01<\frac{(\triangle x)^{2}}{2 M \epsilon \max \left(\gamma+\gamma^{\prime \prime}\right) \operatorname{dim}},
$$

which is derived using the von Neumann stability analysis.

\section{Simulation results for cubic anisotropy}

In Fig. 1, the simulation results referring to the parameters $F_{\alpha \beta}=0.06, F_{\alpha \beta}=0.15$ and $F_{\alpha \beta}=0.45$ in the cubic anisotropy, Eq. (9), are presented in the left, middle, and right columns, respectively. The initial circular inclusion (red dashed lines) and the transitional $t=30$ states are shown in the top row. Herein, the thick black lines correspond to the locus $\phi=0.5$, and the thin black lines emphasize the diffuse interface region, due 

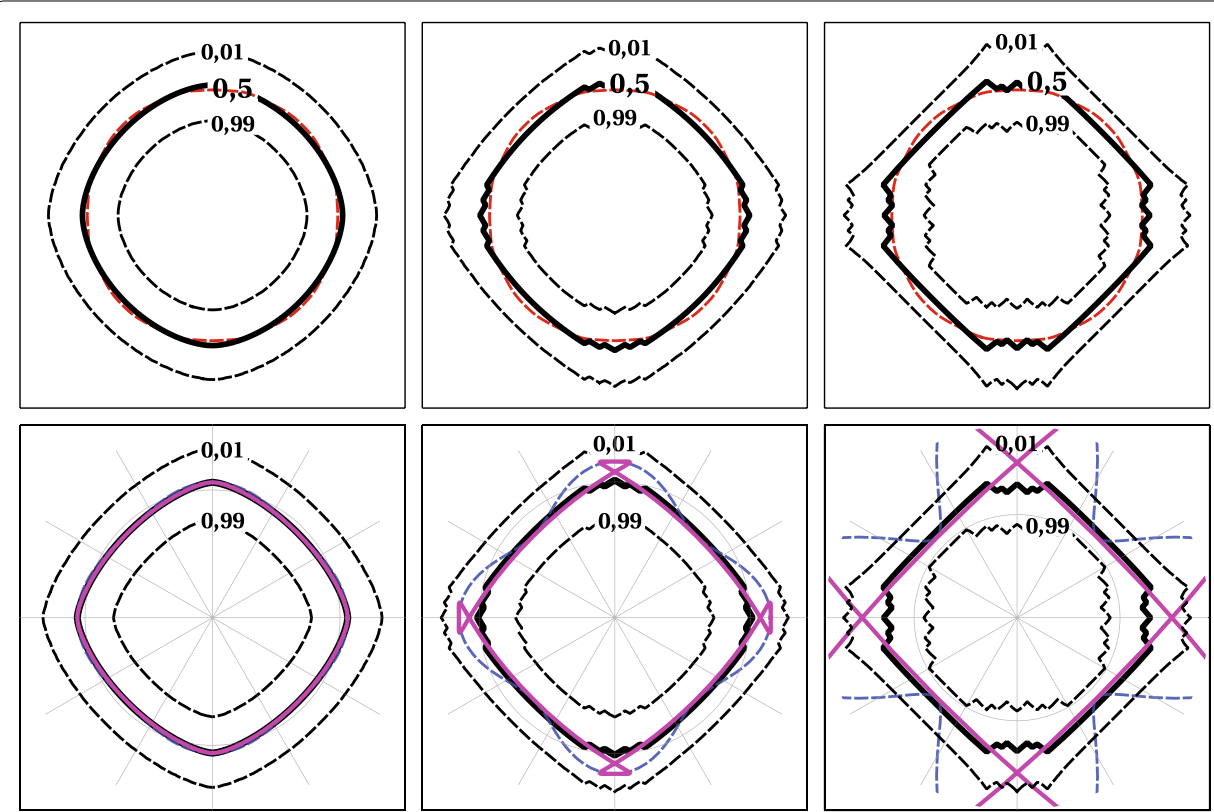

Fig. 1 All graphs show the precipitate in the simulation domain during the evolution, due to the classical model, Eqs. (12) and (13), and for the anisotropy parameter $F_{\alpha \beta}=0.06$ in the left, $F_{\alpha \beta}=0.15$ in the middle, and $F_{\alpha \beta}=0.45$ in the right columns. In the top row, the initial setup $(t=0)$ is shown as a red dashed line, and an intermediate state $(t=30)$ is shown with black solid and dashed lines, corresponding to the loci of $\phi=0.5, \phi=0.01$, and $\phi=0.99$. In the bottom row, the final $(t=600)$ results (black lines) are overlapped by the polar plots of the corresponding Wulff shapes (magenta lines), due to the appropriate interface energy plots (blue lines)

to $\phi=0.01$ and $\phi=0.99$. To compare the equilibrium shape with the simulation results, the corresponding polar plot of the anisotropy function with the appropriate maximal radius, and with the corresponding equilibrium shape overlaps the plot of the inclusion in the simulation domain. Thus, in the bottom row, the numerically stationary states $t=600$ (black lines) as well as the anisotropy plot (blue lines) with the Wulff shapes (magenta lines) are demonstrated.

It is evident from this depiction that in the well-posed problem, the diffuse interface remains smooth during the evolution, whereas instabilities in the form of wrinkles are noticed in the transitional as well as in the final state, for the ill-posed problem. Furthermore, for the well-posed problem, the locus of $\phi=0.5$, in the numerical stationary state, excellently matches the Wulff shape.

In Fig. 2, we show the simulation results with the same simulation setups as in Fig. 1, but we use the natural model, Eq. (15), in the phase-field Eq. (5). An ideal match between the Wulff shape and the simulated result, without any instabilities for weak anisotropic conditions $\left(F_{\alpha \beta}=0.06\right)$, is observed in the left plots of Fig. 2. What is interesting is an excellent match between the predicted and simulated formations for the anisotropy parameter $\left(F_{\alpha \beta}=0.15\right)$, for which the corresponding phase-field model is ill-posed (middle columns). In contrast to the same scenario with the classical model in Fig. 1, the wrinkles which appear during the evolution in the top middle plot vanish in the final state in the plot below. 

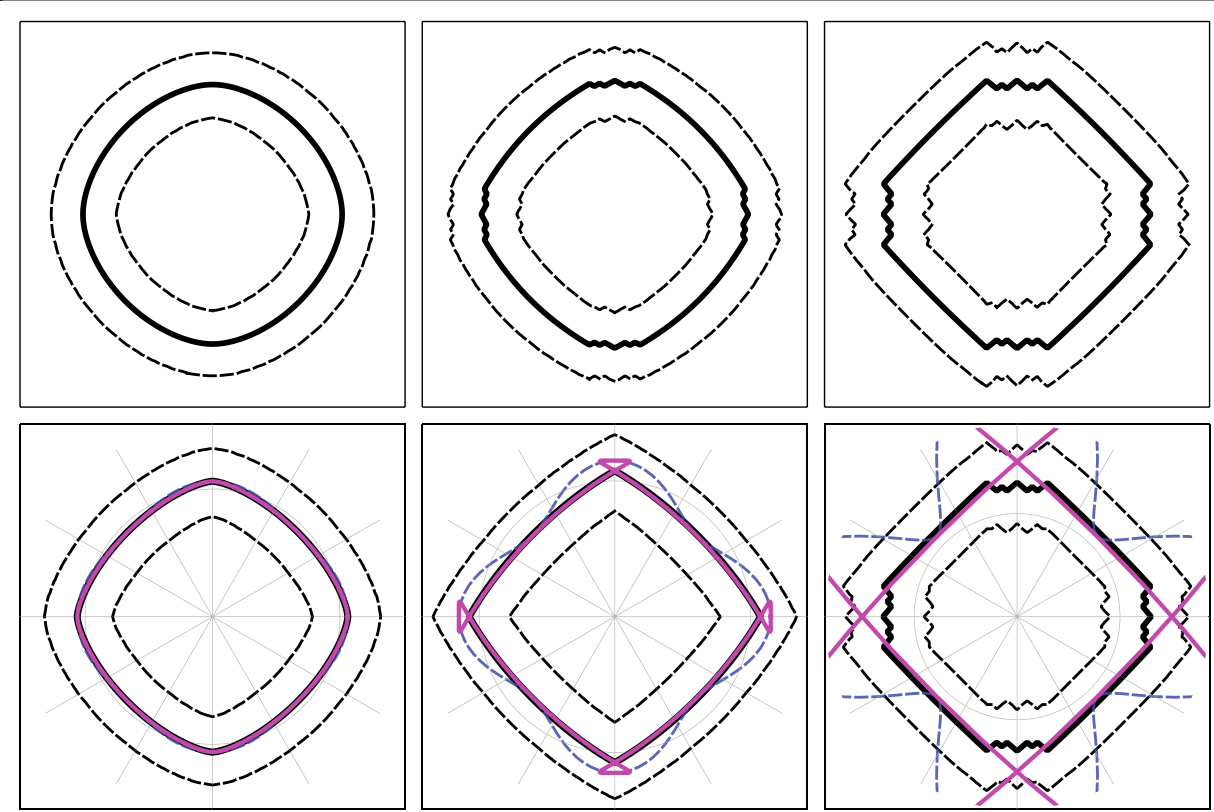

Fig. 2 Parameters as in Fig. 1, but for the natural model, Eqs. (14) and (15)

Considering conditions of strong anisotropies $\left(F_{\alpha \beta}=0.45\right)$ with missing orientations, the final shapes significantly vary from the desired equilibrium shape in both the classical and the natural model. Moreover, the physical instability for the polar angles $\theta$, with $A(\theta)+A^{\prime \prime}(\theta)<0$, results in the non-negligible corrugations in the interface profiles, which occur during the simulation and in the numerically stationary state.

In Fig. 3, we demonstrate the impact of the regularization in the classical model, Eq. (20), in the top rows, and in the natural model, Eq. (21), in the bottom rows, by applying a different scaling in the regularization term, $B=0.06,0.3$, and 0.6 , respectively. Both models behave similarly. Thus, including the curvature contribution to the interfacial energy into the objective free energy functionals, Eqs. (16) and (17), does not regularize the evolution equation, as indicated by the roughened peaks for small coefficients. However, with an increasing weight of the regularization term, the tips of the crystal become more regular. By choosing $B=0.6$, the predicted equilibrium shape is matched. On the other hand, using the explicit Euler scheme for the temporal update of the phase-field value, the temporal step $\Delta t$ depends on the coefficient $B$ and can decrease significantly.

\section{Simulation results for elliptical anisotropy}

The elliptic anisotropy function in Eq. (10) could be of interest to model needle-shaped crystals. As mentioned in the previous paragraph, for the anisotropy parameter $I_{\alpha \beta}>2$, the corresponding phase-field evolution Eq. (5) becomes ill-posed, because of the presence of missing orientations.

In Fig. 4, the results of the virtual experiments are shown for the classical model. As in the preceding simulations for the cubic anisotropy, the initial $(t=0)$ circular precipitate, marked with a red dashed line, is placed in the center of the simulation domain. For different anisotropy strengths, $I_{\alpha \beta}=2$ on the left and $I_{\alpha \beta}=5$. on the right, the 

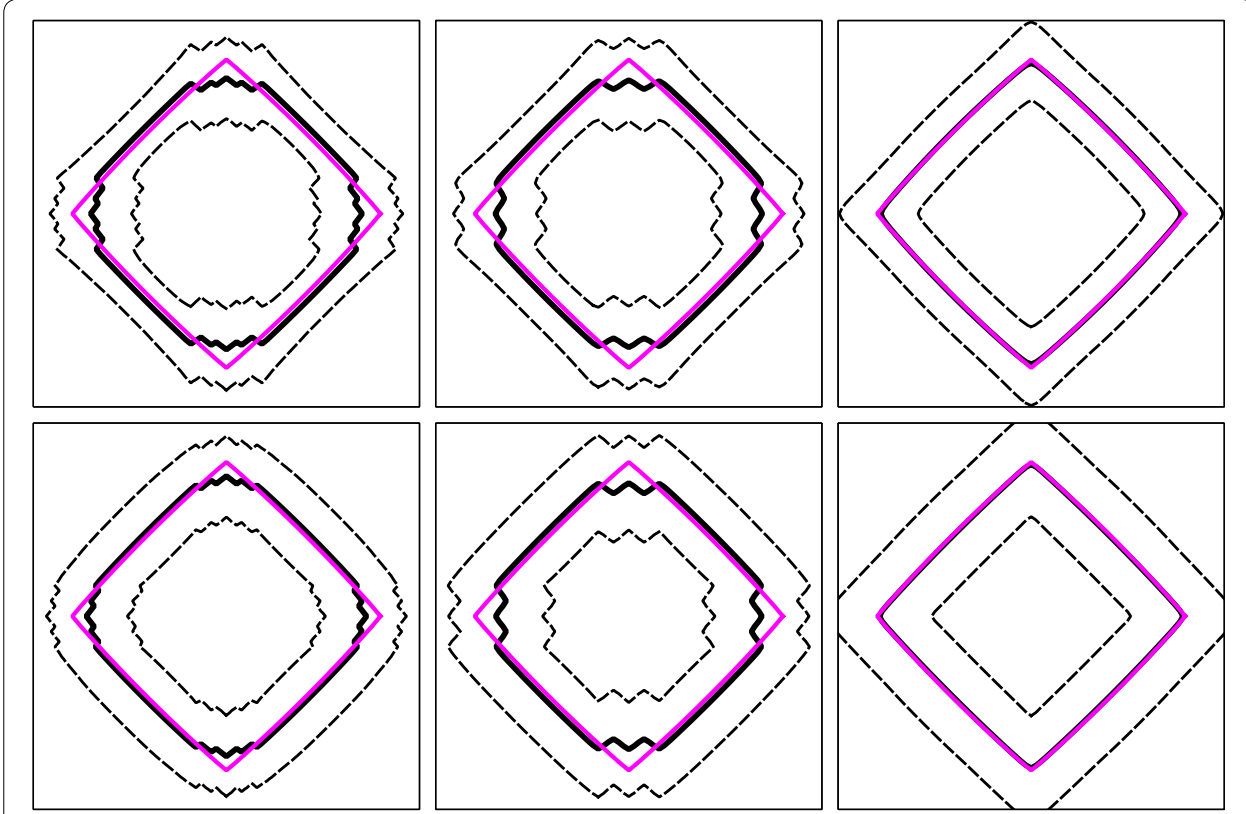

Fig. 3 Equilibrium shape (magenta lines) and final precipitate shapes in the simulation domain (black lines) for the cubic anisotropy, Eq. (9), with $F_{\alpha \beta}=0.45$ for the regularized classical model, Eq. (16), in the top row, and for the regularized natural model, Eq. (17), in the bottom row. In the left, in the middle and in the right columns, the coefficient $B$ in Eqs. (20) and (21) is $0.06,0.3$, and 0.6 , respectively
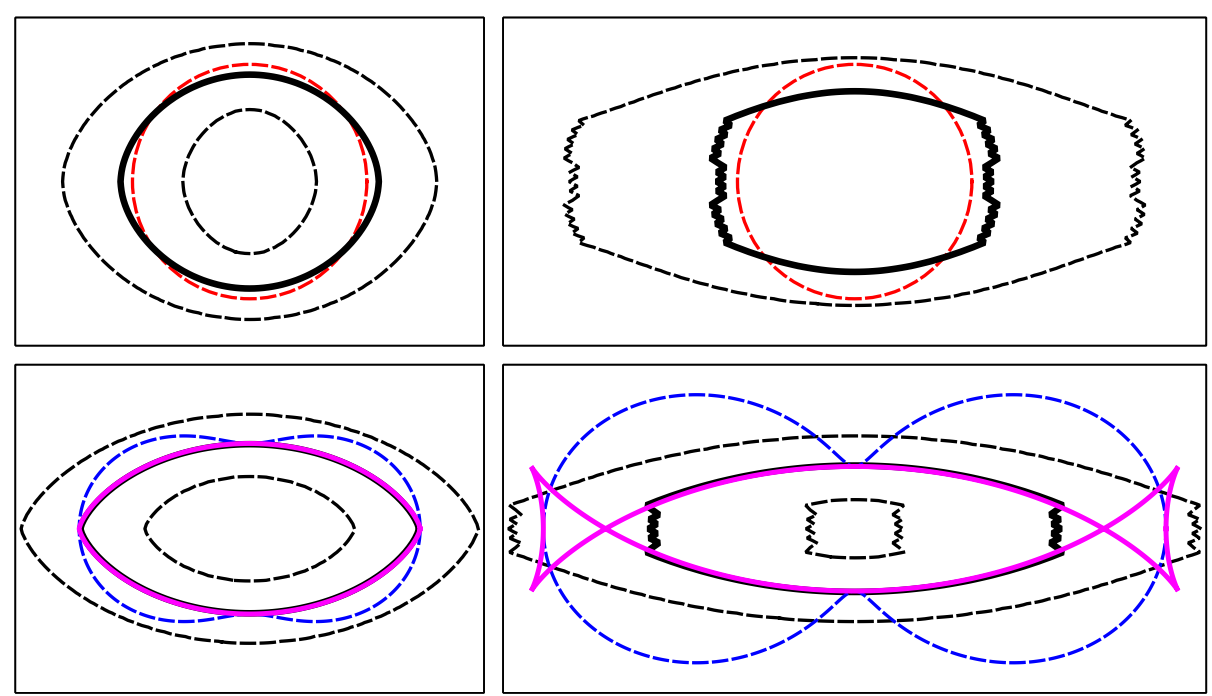

Fig. 4 Evolution of a precipitate, according to the classical model with elliptical anisotropy, with $I_{\alpha \beta}=2$ in the left, and $I_{\alpha \beta}=5$ in the right columns. In the top row, the initial setup $(t=0)$ is shown as a red dashed line, and an intermediate state $(t=30)$ is, respectively, shown with black solid and dashed lines, corresponding to the loci of $\phi=0.5, \phi=0.01$, and $\phi=0.99$. In the bottom row, the final shapes (black lines) are overlapped by the corresponding Wulff shapes (magenta lines), due to the appropriate surface energy plots (blue lines)

intermediate evolution state is shown in the top row. The final simulation states with the referred isolines (black solid and black dashed lines) as well as the aimed Wulff shape (magenta solid line) are shown in the bottom row, as the result of the corresponding anisotropy plot (blue dashed line). 
The intermediate states as well as the final states reveal the well- and ill-posedness of the corresponding phase-field equation, by the presence or absence of the roughness in the diffuse interface. For the simulation with $I_{\alpha \beta}=2$, in the left image of Fig. 4, the diffuse interface is smooth, but with orientation-dependent width. The final shape shows an ideal match with the prescribed equilibrium shape and without any instabilities for weak anisotropic conditions.

For the strong twofold anisotropy with $I_{\alpha \beta}=5$, in the intermediate state $(t=30)$ as well as in the final state, which is shown in the top-right and bottom-right plots of Fig. 4, the interface width extremely depends on the interface orientation. Since the isoline of $\phi=0.99$ fails in the intermediate state, the absence of the bulk cells with $\phi=1$ inside the precipitate is remarkable. Thus, the total inlay is a diffuse interface. During the evolution, the precipitate volume redistributes in the simulation domain, so that the bulk cells appear in the final state, in the bottom-right plot, but the corrugations remain over the simulation time.

The same simulation setup is employed for the natural model, Eq. (15), in the phasefield evolution Eq. (5). The corresponding simulation results are shown in Fig. 5. As for the classical model, the results for the weak anisotropy case, with a well-defined problem, match excellently with the desired equilibrium shape in the left column of Fig. 5. For the ill-posed phase-field evolution equation, both the intermediate as well as the final states are non-physical.

For the regularization, we, respectively, use two different dimensionless corner energies, $B=0.06$ and $B=0.3$, in the modified classical and natural models of Eqs. (16) and (17). In all corresponding numerical experiments, the inclusion evolves with multiple peaks. Using the dimensionless corner energy parameter of $B=0.06$ in the classical model sufficiently regularizes the final shape, which excellently matches with the prescribed equilibrium shape, as displayed in the top-left plot of Fig. 6 . This is not the case for the regularized natural model, as can be seen in the plot below the same figure. Increasing the corner energy to the non-dimensional value of $B=0.3$, the corresponding

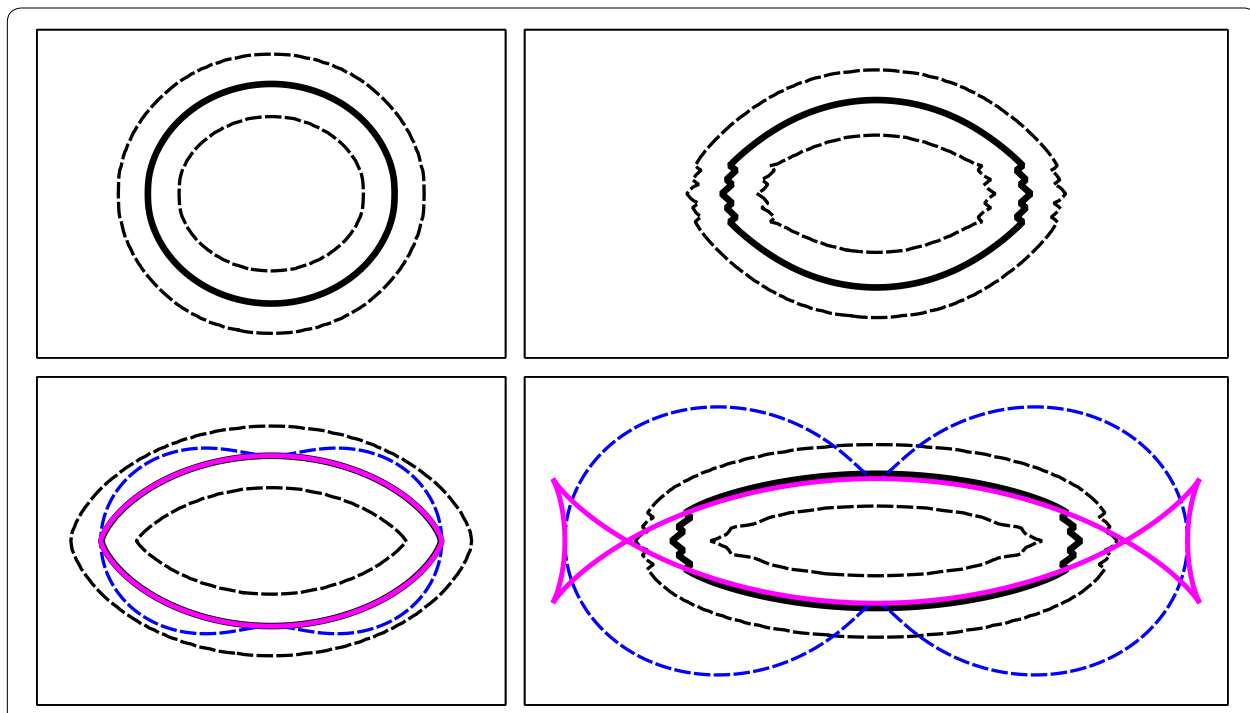

Fig. 5 Parameters as in Fig. 4, but for the natural model 


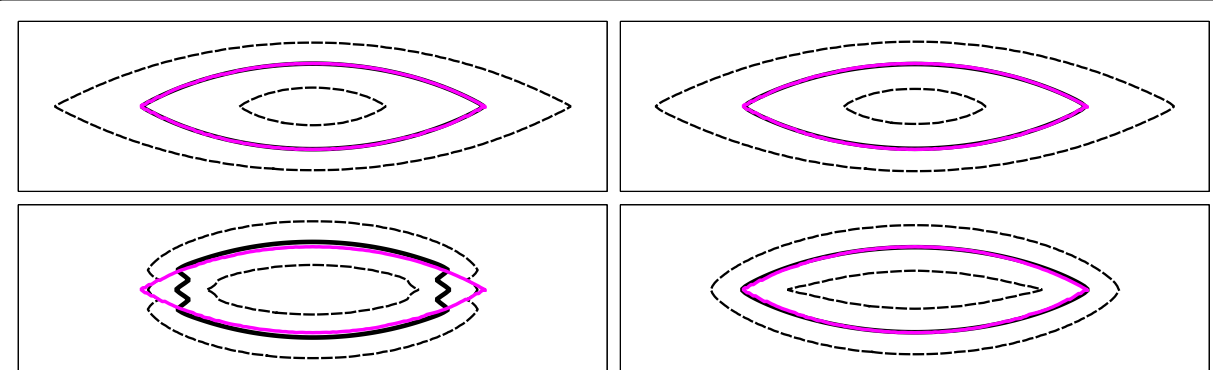

Fig. 6 Equilibrium shape (magenta lines) and final precipitate shapes in the simulation domain (black lines) for the elliptic anisotropy, Eq. (10), with $I_{\alpha \beta}=5$ for the regularized classical model in the top row, and for the regularized natural model in the bottom row. In the left and right columns, the coefficient $B$ is chosen to be 0.06 and 0.3 , respectively

simulation results are demonstrated in the right column of Fig. 6 . Since the diffuse interface width is inhomogeneous for the classical model, the diffuse interface width is independent of the interface orientation, for the natural model, and can be estimated with Eq. (8).

Hereby, the needle-shaped crystals can be modeled and simulated using the elliptical anisotropy. Empirically, we find that the dependence of the ratio of the semi-axes $R$, of the equilibrium lens, on the anisotropy parameter $I_{\alpha \beta}$ is as follows:

$$
R\left(I_{\alpha \beta}\right)=\frac{8}{\sqrt{\pi}} \frac{\Gamma\left(\frac{I_{\alpha \beta}}{5}+\frac{1}{2}\right)}{\Gamma\left(\frac{I_{\alpha \beta}}{5}\right)},
$$

with $\Gamma(x)$ being the gamma function.

\section{Simulation results for crystalline anisotropy}

As mentioned, the crystalline anisotropy in Eq. (11) allows to, respectively, construct arbitrary convex crystals with corners, lines, and planar facets in two and three dimensions. The incorporation of the faceted anisotropy into the classical phase-field model is widely used in the phase-field community, for example by Prajapati et al. (2017), and was discussed in detail in the past by Taylor and Cahn (1998). The difficulty in the application of this anisotropy function lies in its non-differentiability for the directions with corners in the energy polar plots, as indicated by gray vectors in the bottom plots of Fig. 7, which are also perpendicular to the interface of the Wulff shape. Although the derivative does not exist "in the interfacial regions, $\phi$ moves swiftly to approximate its equilibrium profile (in the same manner as when $A$ is differentiable)"-Taylor and Cahn (1998).

In the left and right plots of Fig. 7, we present the simulation results for the classical and natural models, respectively. The red dashed lines in the top plots signify the initial setup, and the black lines refer to the contour lines of the phase-field in an intermediate state $(t=30)$. In the bottom plots, the end states $(t=600)$ of the evolution Eq. (5), with the locus of $\phi=0.5$, are shown with the solid black line, and for $\phi=0.01$ and $\phi=0.99$ with black dashed lines. The anisotropy function and the corresponding equilibrium shape are, respectively, plotted in a polar plot, with dashed blue and solid magenta lines.

As the reader can see in Fig. 7, the phase-field evolution equation for the classical model ideally converges to the prescribed equilibrium shape, whereas double peaks at 

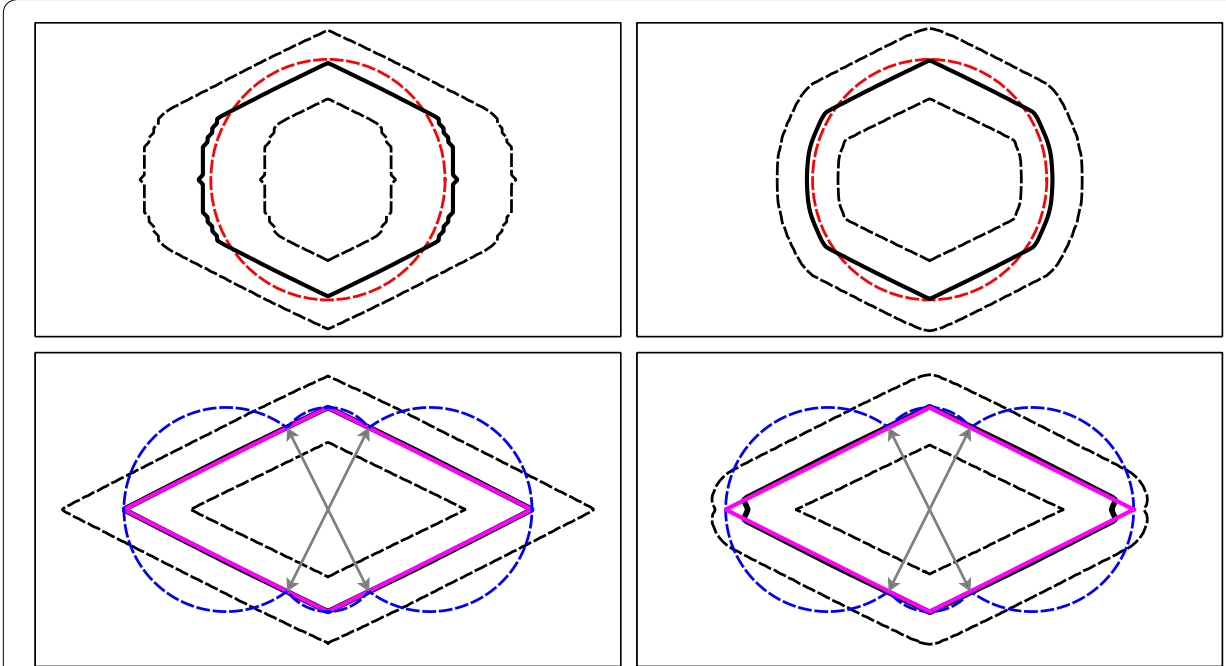

Fig. 7 Precipitate in the simulation domain for the crystalline anisotropy is reflected in the left column, for the classical model, and in the right column, for the natural model. In the top row, the initial setup $(t=0$ ) is shown as a red dashed line, and an intermediate state $(t=30)$ is shown with black solid and dashed lines, corresponding to the loci of $\phi=0.5, \phi=0.01$, and $\phi=0.99$, respectively. In the bottom row, the final $(t=600)$ results (black lines) are overlapped by the corresponding Wulff shapes (magenta lines), due to the appropriate surface energy plots (blue lines)

the sharp corners are observed in crystalline anisotropy, utilizing the natural model, Fig. 7. Introducing the Willmore regularization term to the crystalline anisotropy function, Eq. (11), with previously considered dimensionless corner energy parameters of $B=0.06$ and $B=0.3$, yields the results in Fig. 8. The regularization term corrects the previously observed double peaks for the natural model and renders a good consistency between the resulting and expected structures.
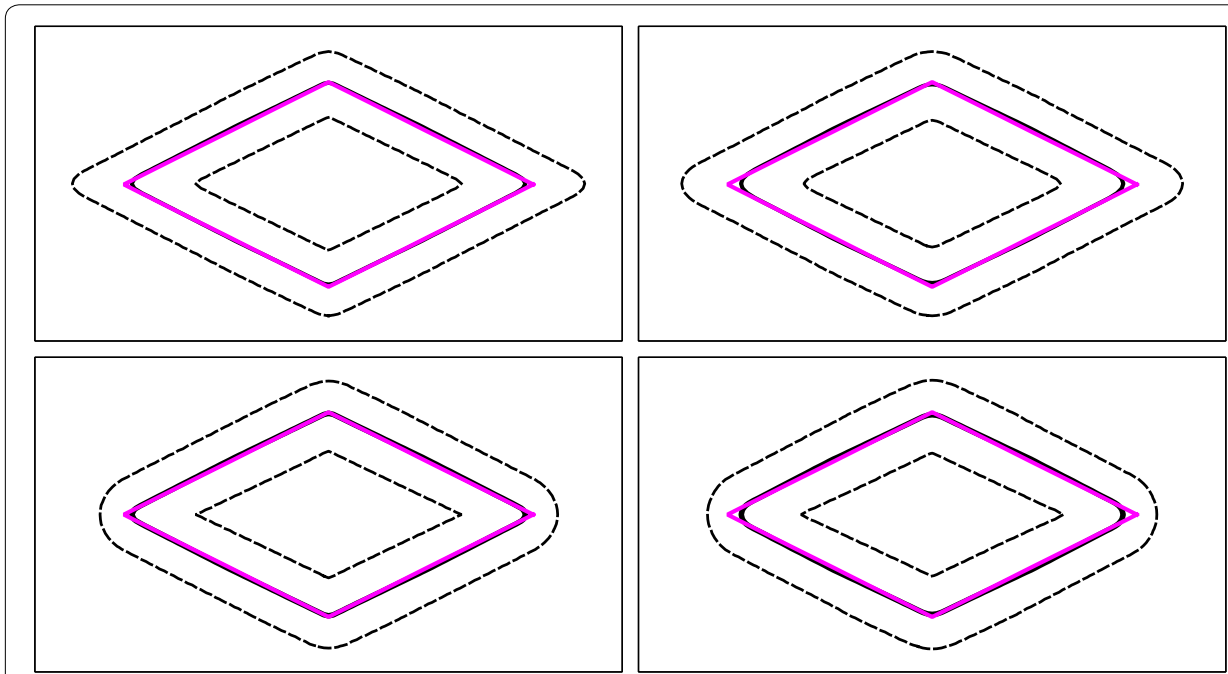

Fig. 8 The final simulation state (black lines) for the crystalline anisotropy function and for the regularized classical model in the top plots, and for the natural model in the bottom plots. On the left, the dimensionless corner energy parameter is $B=0.06$, and $B=0.3$ on the right. The mangenta line signs the equilibrium shape 
Note that for the same dimensionless corner energy parameter, the contour lines of the diffuse interface meet the corner in the sharp interface better for the classical model. For the natural model, the isolines of the phase-field smoothen from the inside to the outside. In spite of the model, the prescribed equilibrium shape is well resolved by the locus of the phase-field profile $\phi=0.5$.

\section{Discussion}

In this work, we analyze the outcomes of the numerical experiments, which are based on three different anisotropy formulations within the context of phase-field modeling. Simulation results for four approaches are discussed, namely: the classical phase-field model, the natural model with the orientation-dependent potential, as well as their Willmore-regularized models, whereby an additional term is incorporated in the objective functional. Built on the presented simulation results, we reveal the applicability and the limitation of every approach, with added focus on the consistency between the numerical equilibrium shape and the corresponding Wulff shape.

As long as the surface energy anisotropy has a non-negative surface stiffness, i.e. $\sigma(\theta)+\sigma^{\prime \prime}(\theta) \geq 0$, the results of the virtual experiments, utilizing the classical model, show an excellent agreement with the predicted Wulff shapes, see the left plots of Figs. 1, 4, and 7. For the smooth anisotropy formulation in Eqs. (9) and (10), and with the well-defined phase-field equation, according to the natural model, the resulting simulated shapes match with the equilibrium formation, see the left plots in Figs. 2 and 5.

When the surface stiffness becomes negative, $\sigma(\theta)+\sigma^{\prime \prime}(\theta)<0$, with respect to the increasing anisotropy strength $F_{\alpha \beta}$ or $I_{\alpha \beta}$, the phase-field evolution equations for both models become ill-posed. The developed corrugations of the diffuse interface indicate the ill-posedness of the mathematical problem, see the right plots of Figs. 1, 2, 4, and 5.

This interface behavior for strong anisotropy functions is well known (Taylor and Cahn 1998; Torabi et al. 2008) and provides a rethinking of the original models (Wheeler 2006; Torabi et al. 2008). Thus, an additional higher order term is incorporated into the objective functional, with the objective to smoothen the zigzags in the interface. Originally, the new term corresponds to the curvature square, and results from the series expansion of the general surface energy density (Torabi and Lowengrub 2012).

As was shown in Figs. 3 and 6, the sole introduction of the regularized model does not guarantee the preservation of the equilibrium shape. Furthermore, the phase-field evolution equation remains ill-posed, since the interface corrugations occur during the simulation as well as in the final state. With an increasing model parameter, $B$, in front of the regularization term, the number of the zigzag peaks decreases during the evolution, while the sharp corners are approximately resolved with a smooth phase-field locus, $\phi=0.5$.

Furthermore, the classical model seems to be more preferable for the anisotropic phase-field models, since it quantitatively resolves the well-posed problems and requires a smaller coefficient in front of the regularization term for the ill-posed configurations, in contrast to the natural model, see Fig. 6 . The reduction of the peaks in the regularized classical models and the remaining corrugation in the natural model in Fig. 6 appear with respect to the differences in the diffuse interface widths in both models. While the diffuse interface in the classical model is orientation-dependent, the diffusional term in 
the phase-field evolution equation is dominating and smoothens the irregularities in the interface. For the natural model, the interface width solely depends on the model parameter $\epsilon$, Eq. (8), and thus, the zigzags remain until the final state.

However, using the classical model, the anisotropy strength strongly affects the diffuse interface width for interfaces with orientations declined from the corner vectors, so that this effect strongly influences the coalescence behavior between two neighboring phases (Ma et al. 2006). Moreover, using the same simulation parameters, but increasing the dimensionless surface energy to $\sigma_{0}=2$, will double the diffuse interface width in every direction, so that only a few bulk cells-if existing at all-are present. Therefore, in the setups with multiple phases, but with interfacial energies of different magnitudes, the diffuse interface width varies significantly, unless the interfacial modeling parameter $\epsilon_{\alpha \beta}$ is used individually for every two-phase interfaces (Tóth et al. 2015). The mentioned drawback is not present in the natural model; thus, the diffuse interface width between two phases in a multiple phase setup remains the same.

\section{Outlook}

In the previous paragraphs, we discussed the advantages and disadvantages of the presented models. An extension of the models in multiphase field contexts, to simulate the grain growth of multiple oriented grains in the Earth's crust, is of general interest for future research. Since the extension of the classical model for multiple phases is widely used (Nestler et al. 2005), the natural multiphase-field model, as well as the regularized multiphase-field models are poorly studied.

\section{Multiphase-field models}

Originating from the multiphase-field model from Nestler et al. (2005), we present a possible extension of the two-phase field model to multiple phases. For the classical multiphase-field model, the objective free energy functional, consisting only of the surface energy terms, is written as follows:

$$
\mathcal{F}_{1}=\int_{V} \sum_{\alpha<\beta}^{N} \gamma_{\alpha \beta}(\epsilon A_{\alpha \beta}^{2}\left(\boldsymbol{q}_{\alpha \beta}\right)\left|q_{\alpha \beta}\right|^{2}+\frac{1}{\epsilon} \underbrace{\frac{16}{\pi^{2}} \phi_{\alpha} \phi_{\beta}}_{w_{\alpha \beta}})+w_{h o}(\boldsymbol{\phi}) \mathrm{d} V
$$

with the generalized asymmetric gradient vector $\boldsymbol{q}_{\alpha \beta}=\phi_{\alpha} \nabla \phi_{\beta}-\phi_{\beta} \nabla \phi_{\alpha}$, and with the higher order term

$$
w_{h o}(\phi)=\frac{1}{\epsilon} \frac{16}{\pi^{2}} \sum_{\alpha<\beta<\delta}^{N} \gamma_{\alpha \beta \delta} \phi_{\alpha} \phi_{\beta} \phi_{\delta}
$$

outside of the parentheses, to suppress the third-phase contributions.

The regularized multiphase-field model was also presented by Ghosh et al. (2015), Ghosh (2015), and we write it as

$$
\mathcal{F}_{1}^{r e g}=\int_{V} \sum_{\alpha<\beta}^{N}\left[\gamma_{\alpha \beta}\left(\epsilon A_{\alpha \beta}^{2}\left(\boldsymbol{q}_{\alpha \beta}\right)\left|q_{\alpha \beta}\right|^{2}+\frac{1}{\epsilon} w_{\alpha \beta}\right)+\frac{B_{\alpha \beta}}{\epsilon} H_{\alpha \beta}^{2}\right]+w_{h o}(\boldsymbol{\phi}) \mathrm{d} V .
$$


In contradiction to the model by Ghosh et al. (2015), in which the mean curvature is calculated by $H_{\alpha \beta}=\nabla \cdot \boldsymbol{q}_{\alpha \beta}$, we prefer the definition originating from Eq. (18), written as follows:

$$
H_{\alpha \beta}=\frac{1}{\epsilon}\left(\frac{\partial w_{\alpha \beta}}{\partial \phi_{\alpha}}-\frac{\partial w_{\alpha \beta}}{\partial \phi_{\beta}}\right)-\epsilon\left(\Delta \phi_{\alpha}-\Delta \phi_{\beta}\right) .
$$

Note that both definitions are only equivalent in a two-phase diffuse interface, but not in multiphase junctions.

Consequently, from the previous functional in Eq. (24), the regularized natural model can be written as follows:

$$
\mathcal{F}_{2}^{r e g}=\int_{V} \sum_{\alpha<\beta}^{N}\left[\gamma_{\alpha \beta} A_{\alpha \beta}\left(\boldsymbol{q}_{\alpha \beta}\right)\left(\epsilon\left|q_{\alpha \beta}\right|^{2}+\frac{1}{\epsilon} w_{\alpha \beta}\right)+\frac{B_{\alpha \beta}}{\epsilon} H_{\alpha \beta}^{2}\right]+w_{h o}(\boldsymbol{\phi}) \mathrm{d} V .
$$

Note that in spite of the model, the usage of the multi-obstacle potential requires the back projection of the numerically integrated phase-field values onto the Gibbs simplex in Eq. (2). Thus, by evaluating $N_{A}$ phase-field evolution equations for locally present phases, the right-hand sides $\left(r h s_{\alpha}\left(\phi^{n}\right)\right)$ of Eq. (4), for all $\alpha \in\left[1, N_{A}\right]$, sum up to zero

$$
\sum_{\alpha=1}^{N_{A}} r h s_{\alpha}\left(\phi^{n}\right)=0
$$

Furthermore, all updated phase-field values $\left(\phi_{\alpha}^{n+1}=\phi_{\alpha}^{n}+M \Delta t r h s_{\alpha}\right)$, with values smaller than zero, are inadmissible and are set to zero. Remaining phase-field values are normalized by their sum.

In future work, three-dimensional simulations of crystal growth, with anisotropy functions, and coupled with solute supersaturation (Ankit 2016) and fluid convection (Selzer 2015), can be derived based on the demonstrated results and on the presented multiphase-field models in Eqs. (24) and (25). While the grain boundary energy is assumed to be isotropic in the previous analysis (Ankit et al. 2013, 2015a, b), the declination of misoriented neighboring grains influences the growth behavior by means of anisotropy, and can be directly incorporated into the extended multiphase-field model in Eqs. (24) and (25). Furthermore, the behavior of the contacting phases in multipoint junctions and their contact angles could depend on the regularization term, as the surface energy density also consists of an additional term. While we only show the equilibrium shapes in this work, by applying the volume-constrained Allen-Cahn equation, the formulation of the kinetic anisotropy for crystal growth can change when using different phase-field models.

\section{Conclusions}

In this work, we present results of virtual experiments based on different phase-field models and apply three different anisotropy functions. The virtual experiments, which are presented in this work, do not reproduce the real process, but are of academic interest, because they highlight the effects on the shape of the crystals for the use of different model formulations. Since the presented findings are well known, the different aspects 
and properties can be found in various publications. We give an overview of the concepts for modeling surface energy anisotropy within the phase-field approach. Finally, we present a natural extension to the multiphase-field model.

\section{Authors' contributions}

OT, AS, and PGKA wrote the manuscript. OT and MS drafted the manuscript. OT and MS implemented the appropriate solvers and the algorithm for the Wulff shape determination. AS conducted the preliminary numerical studies under the supervision of OT and MS, in addition to conceptual discussions with DS and BN. AS produced all the figures. All authors edited the manuscript. All authors read and approved the final manuscript.

\section{Author details}

${ }^{1}$ Institute for Applied Materials (IAM-CMS), Karlsruhe Institute of Technology, Building 30.48, Strasse am Forum 7 , 76131 Karlsruhe, Germany. ${ }^{2}$ Insitute of Materials and Processes, Karlsruhe University of Applied Sciences, Moltkestrasse 30, 76133 Karlsruhe, Germany.

\section{Acknowledgements}

The authors thank the integration initiative in geothermal systems of the Karlsruhe Institute of Technology within the Helmholtz program "renewable energies". We thank the reviewers of our manuscript for their useful and interesting comments.

\section{Competing interests}

The authors declare that they have no competing interests.

Availability of data and materials

All relevant data and material are presented in the main paper.

\section{Consent for publication}

Not applicable.

\section{Ethics approval and consent to participate}

Not applicable.

\section{Publisher's Note}

Springer Nature remains neutral with regard to jurisdictional claims in published maps and institutional affiliations.

Received: 11 April 2017 Accepted: 3 October 2017

Published online: 11 October 2017

\section{References}

Abdeljawad F, Medlin DL, Zimmerman JA, Hattar K, Foiles SM. A diffuse interface model of grain boundary faceting. J Appl Phys. 2016;119(23):235306.

Ankit K, Nestler B, Selzer M, Reichardt M. Phase-field study of grain boundary tracking behavior in crack-seal microstructures. Contrib Mineral Petrol. 2013;166(6):1709-23.

Ankit K, Selzer M, Hilgers C, Nestler B. Phase-field modeling of fracture cementation processes in 3-D. J Pet Sci Res. 2015:4:79

Ankit K, Urai JL, Nestler B. Microstructural evolution in bitaxial crack-seal veins: a phase-field study. J Geophys Res Solid Earth. 2015;120(5):3096-118.

Ankit K. Phase-field modeling of microstructural pattern formation in alloys and geological veins. Doctoral dissertation. Karlsruhe Insitute of Technologies. 2016.

Cahn JW, Hilliard JE. Free energy of a nonuniform system. I. Interfacial free energy. J Chem Phys. 1958;28(2):258-67.

Cahn J, Hoffman D. A vector thermodlnamics for anisotropic surfaces_-I. Curved and faceted surfaces. Acta Metall. 1974:22(10):1205-14.

Chen LQ. Phase-field models for microstructure evolution. Ann Rev Mater Res. 2002;32(1):113-40.

Chen Y, Ye X. Projection onto a simplex. arXiv preprint arXiv:1101.6081. 2011.

Choudhury A, Nestler B. Grand-potential formulation for multicomponent phase transformations combined with thininterface asymptotics of the double-obstacle potential. Phys Rev E. 2012;85(2):021602.

Eggleston JJ, McFadden GB, Voorhees PW. A phase-field model for highly anisotropic interfacial energy. Phys D Nonlinear Phenom. 2001;150(1):91-103.

Eiken J, Böttger B, Steinbach I. Multiphase-field approach for multicomponent alloys with extrapolation scheme for numerical application. Phys Rev E. 2006;73(6):066122.

Emmerich H, Travasso R. Phase-field simulations: materials science meets biology and medicine. Philos Mag. 2011;91(1):1-2.

Fleck M, Mushongera L, Pilipenko D, Ankit K, Emmerich H. On phase-field modeling with a highly anisotropic interfacial energy. Eur Phys J Plus. 2011;126(10):1.

Garcke H, Lam KF, Sitka E, Styles V. A Cahn-Hilliard-Darcy model for tumour growth with chemotaxis and active transport. Math Models Methods Appl Sci. 2016;26(06):1095-148.

Garcke H, Nestler B, Stoth B. On anisotropic order parameter models for multi-phase systems and their sharp interface limits. Phys D Nonlinear Phenom. 1998;115(1):87-108. 
Garcke H, Stoth B, Nestler B. Anisotropy in multi-phase systems: a phase field approach. Interfaces Free Bound. 1999;1 (2):175-98.

Ghosh S. Effects of solid-solid boundary anisotropy on directional solidification microstructures. Doctoral dissertation. Ecole Polytechnqiue. 2015.

Ghosh S, Choudhury A, Plapp M, Bottin-Rousseau S, Faivre G, Akamatsu S. Interphase anisotropy effects on lamellar eutectics: a numerical study. Phys Rev E. 2015;91(2):022407.

Herring C. Some theorems on the free energies of crystal surfaces. Phys Rev. 1951;82(1):87.

Hoffman DW, Cahn JW. A vector thermodynamics for anisotropic surfaces: I. Fundamentals and application to plane surface junctions. Surf Sci. 1972;31:368-88.

Karma A, Rappel WJ. Quantitative phase-field modeling of dendritic growth in two and three dimensions. Phys Rev E. 1998;57(4):4323.

Kobayashi R. Modeling and numerical simulations of dendritic crystal growth. Phys D Nonlinear Phenom. 1993;63(3-4):410-23.

Kobayashi R, Warren JA, Carter WC. Vector-valued phase field model for crystallization and grain boundary formation. Phys D Nonlinear Phenom. 1998;119(3-4):415-23.

Korbuly B, Pusztai T, Tóth Gl, Henry H, Plapp M, Gránásy L. Orientation-field models for polycrystalline solidification: grain coarsening and complex growth forms. J Cryst Growth. 2017:457:32-7.

Landau LD, Lifschitz EM. Lehrbuch der theoretischen Physik IX. Statistische Physik II: Theorie des kondensierten Zustands. Europa-Lehrmittel. 1992.

Lee D, Kim J. Comparison study of the conservative Allen-Cahn and the Cahn-Hilliard equations. Math Comput Simul. 2016;119:35-56.

Ma N, Chen Q, Wang Y. Implementation of high interfacial energy anisotropy in phase field simulations. Scr Mater. 2006;54(11):1919-24.

Makki A. On the viscous Allen-Cahn and Cahn-Hilliard systems with willmore regularization. Appl Math. 2016;61 (6):685-725.

McFadden GB, Wheeler AA, Braun RJ, Coriell SR, Sekerka RF. Phase-field models for anisotropic interfaces. Phys Rev E. 1993:48(3):2016.

Miranville A. On an anisotropic Allen-Cahn system. Cubo (Temuco). 2015;17(2):73-88.

Mullins WW. Proof that the two-dimensional shape of minimum surface free energy is convex. J Math Phys. 1962;3(4):754-9.

Nestler B, Garcke H, Stinner B. Multicomponent alloy solidification: phase-field modeling and simulations. Phys Rev E. 2005;71(4):041609.

Nestler B, Wendler F, Selzer M, Stinner B, Garcke H. Phase-field model for multiphase systems with preserved volume fractions. Phys Rev E. 2008;78(1):011604.

Plapp M, Karma A. Eutectic colony formation: a phase-field study. Phys Rev E. 2002;66(6):061608.

Prajapati N, Selzer M, Nestler B. Computational modeling of calcite cementation in saline limestone aquifers: a phasefield study. Geotherm Energy. 2017;5(1):15.

Schneider D, Tschukin O, Choudhury A, Selzer M, Böhlke T, Nestler B. Phase-field elasticity model based on mechanical jump conditions. Comput Mech. 2015;55(5):887-901.

Sekerka RF. Analytical criteria for missing orientations on three-dimensional equilibrium shapes. J Cryst Growth. 2005;275(1):77-82.

Selzer M. Mechanische und Strõmungsmechanische Topologieoptimierung mit der Phasenfeldmethode. Doctoral dissertation. Karlsruhe Insitute of Technologies. 2014.

Steinbach I. Phase-field models in materials science. Model Simul Mater Sci Eng. 2009;17(7):073001.

Steinbach I, Pezzolla F, Nestler B, SeeSSelberg M, Prieler R, Schmitz GJ, Rezende JL. A phase field concept for multiphase systems. Phys D Nonlinear Phenom. 1996;94(3):135-47.

Steinmetz P, Hötzer J, Kellner M, Dennstedt A, Nestler B. Large-scale phase-field simulations of ternary eutectic microstructure evolution. Comput Mater Sci. 2016;117:205-14.

Takaki T. Phase-field modeling and simulations of dendrite growth. ISIJ Int. 2014;54(2):437-44.

Taylor JE, Cahn JW. Diffuse interfaces with sharp corners and facets: phase field models with strongly anisotropic surfaces. Phys D Nonlinear Phenom. 1998;112(3-4):381-411.

Torabi S, Lowengrub J. Simulating interfacial anisotropy in thin-film growth using an extended Cahn-Hilliard model. Phys Rev E. 2012;85(4):041603.

Torabi S, Lowengrub J, Voigt A, Wise S. A new phase-field model for strongly anisotropic systems. In: Proceedings of the Royal Society of London A: mathematical, physical and engineering sciences. 2009. The Royal Society. p. rspa-2008.

Tóth Gl, Pusztai T, Gránásy L. Consistent multiphase-field theory for interface driven multidomain dynamics. Phys Rev B. 2015;92(18):184105.

Wheeler AA. Phase-field theory of edges in an anisotropic crystal. In: Proceedings of the Royal Society of London A: mathematical, physical and engineering sciences. 2006. Vol. 462, No. 2075, p. 3363-84.

Wulff G. XXV. Zur Frage der Geschwindigkeit des Wachsthums und der auflösung der krystallflächen. Z Kristall Cryst Mater. 1901;34(1--6):449-530. 